\title{
Latin trades in groups defined on planar triangulations
}

\author{
Nicholas J. Cavenagh • Ian M. Wanless
}

Received: 8 August 2008 / Accepted: 4 December 2008 / Published online: 18 December 2008

(C) Springer Science+Business Media, LLC 2008

\begin{abstract}
For a finite triangulation of the plane with faces properly coloured white and black, let $\mathcal{A}_{W}$ be the abelian group constructed by labelling the vertices with commuting indeterminates and adding relations which say that the labels around each white triangle add to the identity. We show that $\mathcal{A}_{W}$ has free rank exactly two. Let $\mathcal{A}_{W}^{*}$ be the torsion subgroup of $\mathcal{A}_{W}$, and $\mathcal{A}_{B}^{*}$ the corresponding group for the black triangles. We show that $\mathcal{A}_{W}^{*}$ and $\mathcal{A}_{B}^{*}$ have the same order, and conjecture that they are isomorphic.

For each spherical latin trade $W$, we show there is a unique disjoint mate $B$ such that $(W, B)$ is a connected and separated bitrade. The bitrade $(W, B)$ is associated with a two-colourable planar triangulation and we show that $W$ can be embedded in $\mathcal{A}_{W}^{*}$, thereby proving a conjecture due to Cavenagh and Drápal. The proof involves constructing a $(0,1)$ presentation matrix whose permanent and determinant agree up to sign. The Smith normal form of this matrix determines $\mathcal{A}_{W}^{*}$, so there is an efficient algorithm to construct the embedding. Contrasting with the spherical case, for each genus $g \geq 1$ we construct a latin trade which is not embeddable in any group and another that is embeddable in a cyclic group.

We construct a sequence of spherical latin trades which cannot be embedded in any family of abelian groups whose torsion ranks are bounded. Also, we show that any trade that can be embedded in a finitely generated abelian group can be embedded in a finite abelian group. As a corollary, no trade can be embedded in a free abelian group.
\end{abstract}

I.M. Wanless's research supported by ARC discovery grant DP0662946.

N.J. Cavenagh · I.M. Wanless $(\bowtie)$

School of Mathematical Sciences, Monash University, Vic 3800, Australia

e-mail: ian.wanless@sci.monash.edu.au

N.J. Cavenagh

e-mail: nicholas.cavenagh@sci.monash.edu.au 
Keywords Latin trade $\cdot$ Bitrade $\cdot$ Latin square $\cdot$ Abelian group · Planar triangulation $\cdot$ Smith normal form $\cdot$ Permanent

\section{Introduction}

This paper demonstrates connections between 2-colourable triangulations of the plane, latin trades, matrices whose permanent and determinant agree up to sign, Smith normal forms and finite abelian groups. It can thus be seen to link topological graph theory, combinatorial designs, linear algebra and group theory.

Latin trades describe the difference between two latin squares of the same order. Such differences are implicitly observed in many different papers, some of them very old. The explicit theory of latin trades arose from a shift in perspective, when the set of differences became an object in its own right. In this sense the earliest article on latin trades is [10], where they are referred to as exchangeable partial groupoids. Later, latin trades became of interest to researchers of critical sets (minimal defining sets of latin squares) $[1,6,16]$. Recently, the theory of latin trades has found intersection with topology [9], geometry [8] and group theory [4]. In particular, latin trades may be interpreted topologically and under certain structural conditions, assigned an integer genus.

In a sense we will make precise in $\S 3$, a latin trade $W$ is embeddable in a group $G$ if you can find a copy of $W$ in the Cayley table of $G$. In certain applications (e.g. $[15,23])$ it is useful to find a latin square which differs only slightly from a group. Finding such examples is equivalent to finding a small trade embedded in the group.

Drápal [8] showed that certain partitionings of integer-sided equilateral triangles into smaller ones imply the existence of latin trades of genus 0 which embed in the group $\mathbb{Z}_{n}$. Cavenagh [3] studied embeddings of 3-homogeneous latin trades (a type of latin trade with genus 1) into abelian groups. These results motivate the question of whether there is any relationship between the genus of a latin trade and its embeddability into a group. Lemma 3 and Lemma 4 combine to show:

Theorem 1 For every $g \geq 1$ there is a latin trade of genus $g$ which cannot be embedded in any group, and another latin trade of genus $g$ which can be embedded in a cyclic group.

By contrast, the trades of genus zero can all be embedded in groups. A major result of this paper is:

\section{Theorem 2 Every spherical latin trade can be embedded in a finite abelian group.}

Moreover, there is an efficient algorithm for constructing what is in some sense the canonical (though not necessarily the minimal) such embedding. As indicated in the abstract, we also establish various properties of spherical trades and the groups that trades can be embedded in.

Theorem 1 and Theorem 2 solve Open problem 8 from [24] (Proposed by Aleš Drápal and Nick Cavenagh), which asked: "Can every separated latin trade be embedded into the operation table of an abelian group? If this is not true in general is it true for spherical latin trades?" 


\section{Definitions}

The following definitions and a more comprehensive survey on latin bitrades may be found in [2].

A latin square $L$ of order $n$ is an $n \times n$ array, with the cells of the array occupied by elements of a set $S$ of $n$ symbols, such that each symbol occurs precisely once in each row and once in each column. If we also index the rows and columns of $L$ by the sets $R$ and $C$, respectively, then $L$ may be thought of as a subset of $R \times C \times S$. Specifically, $(r, c, s) \in L$ if and only if symbol $s$ occurs in row $r$ and column $c$ of the latin square.

It is clear that a Cayley table for a group is a latin square. However, there are latin squares which do not correspond to group tables. In general, a latin square is equivalent to an operation table for a quasigroup. A quasigroup is a set of order $n$ with binary operation $\star$ such that for each $a$ and $b \in Q$, there exist unique elements $x$ and $y \in Q$ such that $a \star x=b$ and $y \star a=b$.

A partial latin square (PLS) of order $n$ is an $n \times n$ array, possibly with empty cells, such that each symbol from $S$ occurs at most once in each row and at most once in each column. Thus any subset of a latin square $L$ is a PLS. The converse, however, is not true, as some PLS have no completion to latin squares of the same order.

Two PLS are said to be isotopic if one may be obtained from the other by relabelling within the sets $R, C$ and $S$. Two PLS are said to be conjugate to each other if one may be obtained from the other by considering them as subsets of $R \times C \times S$ and permuting the coordinates in their triples. Two PLS are said to be in the same main class or species if one may be obtained from the other by a combination of isotopy and conjugation.

We next define latin bitrades (several equivalent definitions may be found in [2]).

Definition 1 A latin bitrade is a pair $(W, B)$ of non-empty PLS such that for each $(r, c, s) \in W$ (respectively, $B)$, there exist unique $r^{\prime} \neq r, c^{\prime} \neq c$ and $s^{\prime} \neq s$ such that:

- $\left(r^{\prime}, c, s\right) \in B$ (respectively, $\left.W\right)$,

- $\left(r, c^{\prime}, s\right) \in B$, (respectively, $\left.W\right)$, and

- $\left(r, c, s^{\prime}\right) \in B$, (respectively, $\left.W\right)$.

The size of $(W, B)$ is equal to $|W|$; i.e. the number of filled cells in $W$ (or, equivalently, in $B$ ). We stress that, in this paper, all PLS (and in particular all trades) are by definition finite.

If $(W, B)$ is a latin bitrade, we may refer to $W$ as a latin trade and $B$ as its disjoint mate. Equivalently, a latin trade is a partial latin square $W$ for which there exists a disjoint mate $B$ such that $(W, B)$ is a latin bitrade. It is possible that a latin trade may have more than one choice of disjoint mate, although we will see in Lemma 12 an important case where there is a unique mate satisfying certain extra conditions.

It is immediate from Definition 1 that any isotopy or conjugate of a latin bitrade is also a latin bitrade and that $(W, B)$ is a latin bitrade if and only if $(B, W)$ is a latin bitrade. If $(W, B)$ is a latin bitrade, it is not hard to see that each non-empty row and each non-empty column must contain at least two symbols, and that each 
symbol occurs at least twice (if at all) within $W$ and $B$. The smallest possible size of a latin bitrade is 4; such latin bitrades correspond to latin subsquares of order 2 and are called intercalates. A latin bitrade $(W, B)$ is said to be connected if there exists no latin bitrades $\left(W^{\prime}, B^{\prime}\right)$ and $\left(W^{\prime \prime}, B^{\prime \prime}\right)$ such that $W^{\prime} \cap W^{\prime \prime}=\emptyset, W=W^{\prime} \cup W^{\prime \prime}$ and $B=B^{\prime} \cup B^{\prime \prime}$.

\subsection{The genus of separated, connected latin bitrades}

In this section we exclude from $R \cup C \cup S$ any rows, columns or symbols that are not used within any triple of a particular latin bitrade and we also specify that $R, C$ and $S$ are pairwise disjoint.

Each row $r$ of a latin bitrade $(W, B)$ defines a permutation $\psi_{r}$ of the symbols in row $r$, where $\psi_{r}(s)=s^{\prime}$ if and only if $(r, c, s) \in W$ and $\left(r, c, s^{\prime}\right) \in B$ for some $c$. If $\psi_{r}$ is a single cycle, then we say that the row $r$ is separated. Similarly, we may classify each column and symbol as being either separated or non-separated. If every row, column and symbol is separated, then we say that the latin bitrade $(W, B)$ is separated.

Any non-separated latin bitrade may be transformed into a separated latin bitrade by a process of identifying each cycle in the permutation with a new row, column or symbol, as shown in the following example.

Example 2.1 In the diagram below, subscripts denote symbols from disjoint mates. The first row $r$ of the latin bitrade $(W, B)$ is non-separated since $\psi_{r}=(14)$ (35). The separated latin bitrade $\left(W^{\prime}, B^{\prime}\right)$ is formed by splitting $r$ into two rows.

\begin{tabular}{|ccccc|}
\hline$\cdot$ & $1_{4}$ & $3_{5}$ & $4_{1}$ & $5_{3}$ \\
$1_{2}$ & $3_{1}$ & $2_{3}$ & $\cdot$ & $\cdot$ \\
$2_{1}$ & $4_{3}$ & $5_{2}$ & $1_{4}$ & $3_{5}$ \\
\hline
\end{tabular}

$(W, B)$

\begin{tabular}{|ccccc|}
\hline$\cdot$ & $1_{4}$ & $\cdot$ & $4_{1}$ & $\cdot$ \\
$\cdot$ & $\cdot$ & $3_{5}$ & $\cdot$ & $5_{3}$ \\
$1_{2}$ & $3_{1}$ & $2_{3}$ & $\cdot$ & $\cdot$ \\
$2_{1}$ & $4_{3}$ & $5_{2}$ & $1_{4}$ & $3_{5}$ \\
\hline
\end{tabular}

$\left(W^{\prime}, B^{\prime}\right)$

Note that the process of separation does not change the size of the latin bitrade, or whether or not it is connected.

Given a separated, connected latin bitrade $(W, B)$, we may construct a triangulation $\mathcal{G}_{W, B}$ whose vertex set is $R \cup C \cup S$ and whose edges are pairs of vertices that occur within some triple of $W$ (or, equivalently, some triple of $B$ ). If we define white and black faces of $\mathcal{G}_{W, B}$ to be the triples from $W$ and $B$ (respectively), then $\mathcal{G}_{W, B}$ is a face 2-colourable triangulation of some surface ([9]).

We next orient the edges of $\mathcal{G}_{W, B}$ so that each white face contains directed edges from a row to a column vertex, a column to a symbol vertex and a symbol to a row vertex. It is immediate that each face (black or white) is labelled coherently; i.e. in the subgraph induced by the vertices of a triangular face, both the out-degree and the in-degree of each vertex are 1 . Thus the surface in which $\mathcal{G}_{W, B}$ is embedded is orientable, and Euler's genus formula must give a non-negative, integer value for the 


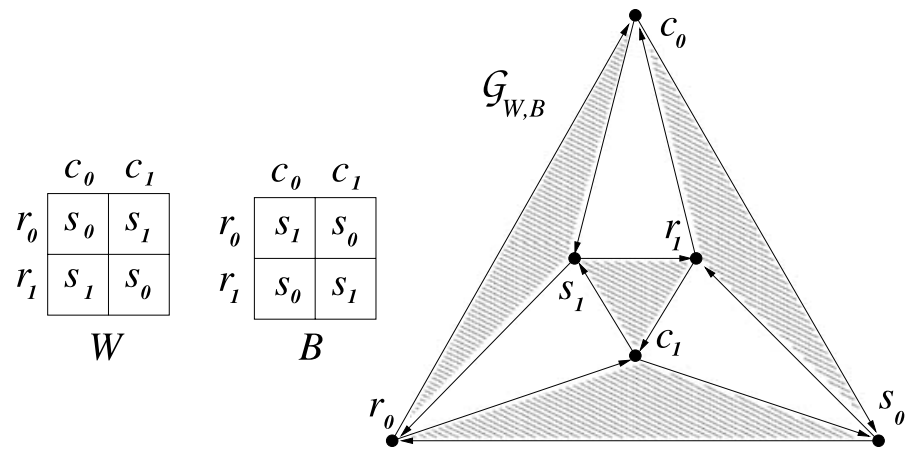

Fig. 1 Intercalates $(W, B)$ with the corresponding triangulation $\mathcal{G}_{W, B}$

genus $g$ :

$$
\begin{aligned}
g & =\frac{1}{2}(2-f+e-v) \\
& =\frac{1}{2}(2-|W|-|B|+3|W|-|R|-|C|-|S|) \\
& =\frac{1}{2}(2+|W|-|R|-|C|-|S|),
\end{aligned}
$$

where $f, e$ and $v$ are the number of faces, edges and vertices of $\mathcal{G}_{W, B}$, respectively. We note that the graph Drápal uses in [9] to calculate the genus is based on the dual of $\mathcal{G}_{W, B}$.

We stress that it only makes sense to calculate the genus of a bitrade if it is separated and connected. Hence, for the remainder of the paper, whenever we ascribe a genus to a bitrade we mean this to imply that the bitrade is separated and connected.

Example 2.2 Figure 1 shows an intercalate latin bitrade $(W, B)$ and its corresponding triangulation $\mathcal{G}_{W, B}$ embedded in the plane. The triangles corresponding to $B$ are shaded. The triangle $\left(r_{0}, c_{0}, s_{0}\right)$ is the external face.

A latin bitrade of genus 0 is called a planar or spherical latin bitrade. We say that a latin trade $W$ is planar or spherical if there exists some disjoint mate $B$ such that the latin bitrade $(W, B)$ is planar. We will see later, in Lemma 12, that such a choice of $B$ is unique, although there may be a trade $B^{\prime} \neq B$ such that $\left(W, B^{\prime}\right)$ is a latin bitrade but is not separated and not connected.

Cavenagh and Lisoněk demonstrated an interesting equivalence involving planar latin bitrades. A planar Eulerian triangulation is an Eulerian graph embedded in the plane with each face a triangle. In the following theorem the latin bitrades are unordered; meaning $(W, B)$ is considered to be equivalent to $(B, W)$.

Theorem 3 ([5]) Planar embeddings of unordered latin bitrades are equivalent to planar Eulerian triangulations. 


\section{Embeddings}

A partial latin square $P$ is embedded in a quasigroup $Q$ with operation $\star$ if $R, C, S \subseteq$ $Q$ and $r \star c=s$ for each $(r, c, s) \in P$. We say that a PLS $P$ embeds in, or is embeddable in, a quasigroup $Q$ if it is isotopic to a PLS which is embedded in $Q$. If $Q$ is a group, this property is species invariant, as shown in the following lemma.

Lemma 1 Let $G$ be an arbitrary group and $P, P^{\prime}$ two PLS from the same species. Then $P$ embeds in $G$ iff $P^{\prime}$ embeds in $G$.

Proof By definition, embedding is isotopy invariant, so it suffices to consider the case where $P$ and $P^{\prime}$ are conjugate. This case follows directly from Theorem 4.2.2 of [7], which states that the six conjugates of the Cayley table of a group are isotopic to each other. So if $P$ embeds in $G$ we take conjugates of the resulting embedding to yield embeddings for the different conjugates of $P$.

Lemma 1 is not true, in general, for embeddings in Latin squares other than group tables. In the following example, $P$ embeds in $L$ as shown by the entries in bold, but the transpose of $P$ cannot be embedded in $L$.

$$
P=\begin{array}{lll}
0 & 1 & 2 \\
1 & 2 & 0
\end{array} \quad L=\begin{array}{llllll|}
0 & 1 & 2 & 3 & 4 & 5 \\
\mathbf{1} & 0 & 3 & \mathbf{2} & \mathbf{5} & 4 \\
\mathbf{2} & 4 & 0 & \mathbf{5} & \mathbf{1} & 3 \\
3 & 5 & 1 & 4 & 2 & 0 \\
4 & 3 & 5 & 1 & 0 & 2 \\
5 & 2 & 4 & 0 & 3 & 1 \\
\hline
\end{array}
$$

Our second lemma shows that embeddability in a group can be tested by considering a restricted class of isotopies.

Lemma 2 Let $P$ be a PLS and suppose that $(r, c, s) \in P$. Let $G$ be a group with identity element $\varepsilon$. If $P$ embeds in $G$ then $P$ is isotopic to a PLS in $G$ by an isotopy that maps each of $r, c$ and $s$ to $\varepsilon$.

Proof Suppose $P$ is embedded in $G$. Define the permutations $I_{1}, I_{2}, I_{3}: G \mapsto G$ by $I_{1}(x)=r^{-1} x, I_{2}(x)=x c^{-1}$ and $I_{3}(x)=r^{-1} x c^{-1}$. The isotopy $\left(I_{1}, I_{2}, I_{3}\right)$ maps $(r, c, s)$ to $\left(r^{-1} r, c c^{-1}, r^{-1} r c c^{-1}\right)=(\varepsilon, \varepsilon, \varepsilon)$.

In this paper we are not concerned with counting the number of ways that a trade can be embedded in a group. However, we make the following observation in passing. Given a trade $W$ and group $G$ it is possible for $W$ to have "essentially different" embeddings $E_{1}$ and $E_{2}$ in $G$ in the sense that there is no isotopism that preserves $G$ and maps $E_{1}$ to $E_{2}$. For example, the order two subgroups of $\mathbb{Z}_{2} \oplus \mathbb{Z}_{4}$ generated by, respectively, $(0,2)$ and $(1,0)$ are "essentially different" embeddings of intercalates. The former is contained in an embedding of $\mathbb{Z}_{4}$, while the latter is not. 
We remind the reader that not every latin square is a Cayley table for a group. A test for whether a latin square is isotopic to a group is the quadrangle criterion. A latin square $L$ satisfies the quadrangle criterion if and only if for each $r_{1}, r_{2}, r_{1}^{\prime}, r_{2}^{\prime}, c_{1}, c_{2}, c_{1}^{\prime} c_{2}^{\prime}, s_{1}, s_{2}, s_{3}$ such that

$$
\left(r_{1}, c_{1}, s_{1}\right),\left(r_{1}, c_{2}, s_{2}\right),\left(r_{2}, c_{1}, s_{3}\right),\left(r_{1}^{\prime}, c_{1}^{\prime}, s_{1}\right),\left(r_{1}^{\prime}, c_{2}^{\prime}, s_{2}\right),\left(r_{2}^{\prime}, c_{1}^{\prime}, s_{3}\right) \in L,
$$

the cells $\left(r_{2}, c_{2}\right)$ and $\left(r_{2}^{\prime}, c_{2}^{\prime}\right)$ contain the same symbol. A latin square is isotopic to the Cayley table of a group if and only if it satisfies the quadrangle criterion (see [7] for a proof).

Observe that if a partial latin square $P$ contains filled cells that fail the quadrangle criterion and if $P$ is a subset of a latin square $L$, then $L$ also fails the quadrangle criterion. Thus if a latin trade fails the quadrangle criterion, it does not embed into any group $G$. However, the converse is not true, as shown by the following example.

Example 3.1 The following latin bitrade $(W, B)$ is separated and connected. It does not fail the quadrangle criterion, as can easily be seen by noting that no pair of columns have more than one symbol in common.

$$
W=\begin{array}{lllllllll}
1 & 2 & 3 & 4 & 5 & 6 & 7 & 8 & 9 \\
2 & 3 & 4 & 5 & 6 & 7 & 8 & 9 & 1 \\
4 & 5 & 9 & 8 & 1 & 3 & 2 & 6 & 7
\end{array} \quad B=\begin{array}{lllllllll|}
2 & 3 & 4 & 5 & 6 & 7 & 8 & 9 & 1 \\
4 & 5 & 9 & 8 & 1 & 3 & 2 & 6 & 7 \\
1 & 2 & 3 & 4 & 5 & 6 & 7 & 8 & 9 \\
\hline
\end{array}
$$

However $W$ cannot be embedded into any group. To see this, take pairs of rows and interpret them as permutations in two row format. Writing these in cycle format, the first two rows give us $\pi_{12}=(123456789)$ whilst the first and third rows give us $\pi_{13}=(148639725)$. If $W$ could be embedded in a group then, applying a condition due to Suschkewitsch [19] related to the proof of Cayley's theorem, we would find that $\pi_{12}$ and $\pi_{13}$ have to generate a group of order at most 9 . As this clearly is not the case, $W$ cannot be embedded in a group.

Another way to produce a latin rectangle that cannot be embedded in any group is to construct one which has rows $i$ and $j$ for which $\pi_{i j}$ is not a regular permutation (a regular permutation is one in which all cycles have the same length).

As the above example demonstrates, the quadrangle criterion cannot be used to prove that a latin trade embeds in some group. However, it is useful for showing that a trade does not embed in any group:

Example 3.2 The smallest connected, separated trade which does not embed in any group is shown below, with its disjoint mate (which also does not embed in a group).
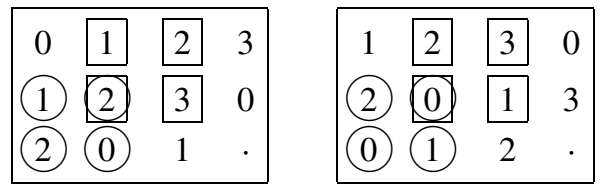
The fact that both trades fail the quadrangle criterion, and thus do not embed in a group, is demonstrated by the two quadrangles marked with $\square$ and $\bigcirc$. The fact that no smaller trade fails to embed in a group is guaranteed by Theorem 2 , since by [20] the only smaller trades of positive genus are isotopic to $\mathbb{Z}_{3}$ and hence (trivially) embeddable in a group.

On the other hand, there are many classes of latin trades which do embed in groups. An early result by Drápal [8] gave a class of geometrically constructed latin trades which embed in $\mathbb{Z}_{n}$ :

Theorem 4 (Drápal, [8]) Let $m$ and $n$ be positive integers. Suppose that we can partition an equilateral triangle of side $n$ into $m$ smaller (integer-sided) equilateral triangles, such that each vertex of a triangle occurs as the vertex of at most 3 of the smaller triangles. Then there exists a corresponding planar latin bitrade $(W, B)$ of size $m$ such that $W$ embeds into $\mathbb{Z}_{n}$.

The fact that the latin trades in the previous theorem are planar led to the exploration of the relationship between the genus of a latin trade and whether it embeds into a group. This is part of the motivation for us to prove that any spherical latin trade is embeddable into an abelian group (Theorem 2).

We spend the rest of this section putting this theorem into context by exploring what happens when the conditions are relaxed or modified. We first show that there is not an analogous result for any genus $g>0$. We then demonstrate an example of each of the following: a latin trade which embeds in an abelian group but not in any cyclic group, a latin trade which embeds in a non-abelian group but not in any abelian group, and a latin trade which does not embed in any abelian group even though it separates to produce a spherical trade. Finally, we consider the case of embeddings into abelian groups which are infinite but finitely generated. We show that this case, in essence, reduces to the finite one.

To see that planarity is a necessary condition for our result, we present the following two lemmas, which together prove Theorem 1.

Lemma 3 For each integer $g>0$, there exists a separated, connected latin bitrade $(W, B)$ of genus $g$ such that $W$ embeds in $\mathbb{Z}_{2 g+1}$.

Proof To construct such a trade, let $W$ be the first three rows of $\mathbb{Z}_{2 g+1}$. Then form $B$ by permuting the rows of $W$ by shifting the top row to the bottom. Clearly $(W, B)$ is a latin bitrade. Since each column has three symbols and each symbol occurs three times, the columns and symbols are separated.

As each symbol $s$ is replaced by symbol $s+1(\bmod 2 g+1)$ in the first two rows, these rows are separated. In the last row, $s$ is replaced by $s-2(\bmod 2 g+1)$. As $2 g+1$ is odd, this row is also separated. Thus we have a separated, connected latin bitrade, so its genus is well-defined. From (1), the genus is

$$
\frac{1}{2}(2+|B|-|R|-|C|-|S|)=\frac{1}{2}(2+3(2 g+1)-3-2(2 g+1))=g .
$$


Lemma 4 For each integer $g>0$, there exists a separated, connected latin bitrade $(W, B)$ of genus $g$ such that $W$ does not embed in any group.

Proof Example 3.2 suffices for $g=1$, so we suppose that $g \geq 2$. Let $S=\{0,1,2\} \times$ $\{0,1,2, \ldots, 2 g\} \backslash\{(2,1),(2,2),(2,2 g)\}$. Define a partial latin square $W$ so that:

$$
W=\{(2,1,4),(2,2,1),(2,2 g, 3)\} \cup\{(i, j, i+j) \mid(i, j) \in S\},
$$

with $i+j$ evaluated modulo $2 g+1$. Next, form $B$ by shifting the top row of $W$ to the bottom. For illustration, we show the pair $(W, B)$ when $g=3$ :

$$
W=\begin{array}{llllllll|}
\hline 0 & 1 & 2 & 3 & 4 & 5 & 6 \\
1 & 2 & 3 & 4 & 5 & 6 & 0 \\
1 & 2 & 4 & 1 & 5 & 6 & 0 & 3 \\
2 & 4 & 1 &
\end{array}
$$$$
B=\begin{array}{lllllll|}
1 & 2 & 3 & 4 & 5 & 6 & 0 \\
2 & 4 & 1 & 5 & 6 & 0 & 3 \\
0 & 1 & 2 & 3 & 4 & 5 & 6 \\
\hline
\end{array}
$$

It is not hard to show that $(W, B)$ is a connected, separated latin bitrade. The calculation that $(W, B)$ has genus $g$ is the same as in the previous lemma. Moreover, $W$ cannot embed in a group as it fails the quadrangle criterion, as shown by the quadrangles displayed in the example above, which are present for all $g \geq 2$.

\subsection{Some contextual examples}

In this subsection, we give some contextual examples. In a number of cases we claim that the example we cite is the smallest possible. All such claims are based on the enumeration of trades and bitrades up to size 19, reported in [20] (the data is downloadable from [22]). Note, by Lemma 1, that it suffices in all cases just to consider one representative of each species.

In our next example, and a number of later examples, we establish that a given trade $W$ cannot be embedded in a certain type of group. We do this by assuming it is embedded in such a group then deriving a contradiction. To aid our calculations we adopt the convention that the rows and columns, respectively, of $W$ are labelled $r_{0}, r_{1}, r_{2}, \ldots$ and $c_{0}, c_{1}, c_{2}, \ldots$ in the order that we give them in the example. Also, if the example contains symbols $0,1,2, \ldots$, then we refer to these in our calculations as $s_{0}, s_{1}, s_{2}, \ldots$, respectively.

Example 3.3 The smallest separated, connected bitrade $(W, B)$ such that $W$ cannot be embedded in any cyclic group has size 10 . The unique (up to interchanging $W$ and $B$ ) example of that size is spherical. It is:

$$
\left.W=\begin{array}{cccc}
0 & 1 & 2 & 3 \\
1 & 0 & \cdot & . \\
2 & \cdot & 1 & . \\
\cdot & 3 & \cdot & 0
\end{array}\right] \quad B=\left[\begin{array}{cccc}
2 & 3 & 1 & 0 \\
0 & 1 & . & \cdot \\
1 & . & 2 & \cdot \\
\cdot & 0 & \cdot & 3
\end{array}\right.
$$

Suppose $W$ embeds in a cyclic group of order $m$ with operation $\circ$ and identity 0 . Assuming, on the basis of Lemma 2, that $r_{0}=c_{0}=s_{0}=0$ we then deduce that $c_{1}=$ 
$0 \circ c_{1}=s_{1}=r_{1} \circ 0=r_{1}$ and $c_{2}=0 \circ c_{2}=s_{2}=r_{2} \circ 0=r_{2}$. Since $2 c_{1}=r_{1} \circ c_{1}=0$ and $c_{1} \neq c_{0}=0$ we conclude that $c_{1}=m / 2$. Now $2 c_{2}=r_{2} \circ c_{2}=s_{1}=c_{1}$ so $c_{2}= \pm m / 4$. Also $r_{3} \circ c_{3}=0$ so $r_{3}=-c_{3}$, but $r_{3} \circ c_{1}=0 \circ c_{3}=c_{3}=-r_{3}$ so $2 r_{3}=-c_{1}=m / 2$. Thus $r_{3}= \pm m / 4$. However this means that $c_{2}, c_{3}$ and $r_{3}$ are each equal to $\pm m / 4$. This is impossible given that $r_{3}=r_{3} \circ c_{0}$ is distinct from both $r_{3} \circ c_{1}=c_{3}$ and $r_{2} \circ c_{0}=c_{2}$, and $c_{2}$ and $c_{3}$ are also distinct from each other.

A similar argument shows that $B$ cannot embed in a cyclic group of order $m$. We deduce in turn that $r_{0}=c_{0}=s_{2}=0, r_{2}=c_{2}=m / 2, r_{3} \circ c_{1}=c_{3}$ and $r_{3} \circ c_{3}=c_{1}$. Combining these last two equations we get $2 r_{3}=0=2 r_{0}=2 r_{2}$, which contradicts the fact that $r_{0}, r_{2}$ and $r_{3}$ are distinct.

Interestingly, although Theorem 2 tells us that $B$ can be embedded in an abelian group (in fact both $B$ and $W$ embed in $\mathbb{Z}_{2} \oplus \mathbb{Z}_{4}$ ), the smallest group that $B$ embeds in is non-abelian. This follows from the above observation that it does not embed in a cyclic group, the obvious fact that it does not embed in $\mathbb{Z}_{2} \oplus \mathbb{Z}_{2}$, and the following embedding in $S_{3}$ :

\begin{tabular}{|llllll|}
\hline $\mathbf{0}$ & $\mathbf{1}$ & $\mathbf{2}$ & $\mathbf{3}$ & 4 & 5 \\
$\mathbf{1}$ & 0 & 3 & $\mathbf{2}$ & 5 & 4 \\
$\mathbf{2}$ & 4 & $\mathbf{0}$ & 5 & 1 & 3 \\
3 & 5 & 1 & 4 & 0 & 2 \\
4 & 2 & 5 & 0 & 3 & 1 \\
5 & $\mathbf{3}$ & 4 & $\mathbf{1}$ & 2 & 0 \\
\hline
\end{tabular}

Example 3.4 The smallest connected separated bitrade $(W, B)$ in which $W$ embeds in a group but not in any abelian group has size 14 . An example of this size is:

\begin{tabular}{|ll|lllll|}
\hline $\mathbf{0}$ & $\mathbf{1}$ & $\mathbf{2}$ & 3 & $\mathbf{4}$ & $\mathbf{5}$ \\
$\mathbf{1}$ & $\mathbf{1}$ & $\mathbf{0}$ & 4 & 5 & $\mathbf{2}$ & 3 \\
$\mathbf{2}$ & $\mathbf{5}$ & $\mathbf{0}$ & 4 & 3 & 1 \\
3 & 4 & 5 & 0 & 1 & 2 \\
4 & 3 & 1 & 2 & 5 & 0 \\
$\mathbf{5}$ & 2 & 3 & 1 & $\mathbf{0}$ & $\mathbf{4}$ \\
\hline
\end{tabular}

\begin{tabular}{|cccccc|}
\hline 1 & 5 & 0 &. & 2 & 4 \\
2 & 1 &. &. & 0 &. \\
5 & 0 & 2 &. &. &. \\
. &. &. &. &. &. \\
. &. &. &. &. &. \\
0 &. &. &. & 4 & 5 \\
\hline
\end{tabular}

The left hand square is the non-abelian group $S_{3}$, in which the trade $W$ is shown in bold. The disjoint mate $B$ is given in the right hand square. To see that there is no smaller example, it suffices by Theorem 2 to check the 33 smaller species of genus 1. These include Example 5.1, 6 bitrades in which both trades are embeddable in abelian groups and 26 bitrades in which both trades fail to embed in any group (as can be shown using the quadrangle criterion).

For the sake of contradiction, assume that $W$ can be embedded in an abelian group with operation $\circ$ and identity 0 . Assuming, on the basis of Lemma 2, that $r_{0}=c_{0}=$ $s_{0}=0$ we then deduce that $c_{1}=0 \circ c_{1}=s_{1}=r_{1} \circ 0=r_{1}$ and $c_{2}=0 \circ c_{2}=s_{2}=$ $r_{2} \circ 0=r_{2}$. Now observe that the two displayed quadrangles force $c_{1} \circ r_{2}=r_{1} \circ c_{2}=$ $s_{4} \neq s_{5}=r_{2} \circ c_{1}$, which violates the assumption that $\circ$ is abelian. 
Example 3.5 The (implicit) condition in Theorem 2 that the trade needs to be separated cannot be abandoned. Consider the following example:
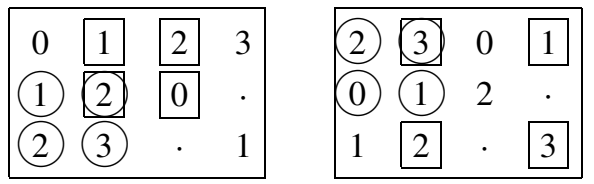

This connected bitrade is not separated since $\psi_{0}=(02)(13)$, although it may be separated into a spherical latin bitrade via the process outlined in $\$ 2.1$. Both trades fail the quadrangle criterion (as demonstrated by the quadrangles shown) and hence cannot be embedded in any group.

\subsection{Embeddings in infinite groups}

Next, we examine embeddings into infinite, not necessarily abelian, groups. The general theme is that if a trade embeds in an infinite group then it usually embeds in a related "smaller" group. We remind the reader that in this paper trades are finite by definition.

Lemma 5 Suppose $H$ is a normal subgroup of $G$ and $\phi$ is the natural homomorphism $\phi: G \mapsto G / H$. Let $P$ be any PLS embedded in $G$. If there exist $k_{1}, k_{2} \in G$ such that $\phi(x)=k_{1} H$ and $\phi(y)=k_{2} H$ for all triples $(x, y, z) \in P$, then $P$ embeds in $H$.

Proof Consider the isotopy which maps each $(x, y, z) \in P$ to

$$
\left(k_{2}^{-1} k_{1}^{-1} x k_{2}, k_{2}^{-1} y, k_{2}^{-1} k_{1}^{-1} z\right) .
$$

This is a well defined isotopy since $k_{1}$ and $k_{2}$ are fixed. It provides another embedding of $P$ in $G$ because

$$
\left(k_{2}^{-1} k_{1}^{-1} x k_{2}\right)\left(k_{2}^{-1} y\right)=k_{2}^{-1} k_{1}^{-1} x y=k_{2}^{-1} k_{1}^{-1} z .
$$

Moreover, $\phi\left(k_{2}^{-1} k_{1}^{-1} x k_{2}\right)=\phi\left(k_{1}\right)^{-1} \phi(x)=H$ and $\phi\left(k_{2}^{-1} y\right)=\phi\left(k_{2}\right)^{-1} \phi(y)=H$ so we have an embedding of $P$ in $H$.

Lemma 6 Suppose $G$ is a group and $\phi: G \mapsto \mathbb{Z}$ a homomorphism. Let $(W, B)$ be a connected latin bitrade such that $W$ is embedded in $G$. Then $W$ embeds in the kernel of $\phi$.

Proof Define

$$
\begin{aligned}
s & =\max \{\phi(z):(x, y, z) \in W\}, \\
r & =\max \{\phi(x):(x, y, z) \in W, \phi(z)=s\}, \\
R & =\{x: \exists(x, y, z) \in W, \phi(x)=r, \phi(z)=s\}, \\
C & =\{y: \exists(x, y, z) \in W, \phi(x)=r, \phi(y)=s-r\} .
\end{aligned}
$$


Let $W^{\prime}$ and $B^{\prime}$ be the restriction of $W$ and $B$, respectively, to the array defined by $R \times C$. Consider $(x, y, z) \in W$. Then $(x, y, z) \in W^{\prime}$ if and only if $\phi(x)=r$ and $\phi(z)=s$. On the other hand, consider $\left(x, y^{\prime}, z\right) \in B$ such that $\phi(x)=r$ and $\phi(z)=$ $s$. By Definition 1 there exist $x^{\prime}, z^{\prime}$ such that $W$ contains the triples $\left(x^{\prime}, y^{\prime}, z\right)$ and $\left(x, y^{\prime}, z^{\prime}\right)$. By our choice of $r, \phi\left(y^{\prime}\right)=\phi(z)-\phi\left(x^{\prime}\right) \geq s-r$ and by our choice of $s$, $\phi\left(y^{\prime}\right)=\phi\left(z^{\prime}\right)-\phi(x) \leq s-r$. Hence $\phi\left(y^{\prime}\right)=s-r$ and $y^{\prime} \in C$. Thus if $\left(x, y^{\prime}, z\right) \in B$, $\phi(x)=r$ and $\phi(z)=s$, then $\left(x, y^{\prime}, z\right) \in B^{\prime}$.

By Definition 1 , for each $(x, y, z) \in W^{\prime}$, there is a unique $y^{\prime} \neq y$ such that $\left(x, y^{\prime}, z\right) \in B$. From above, each such $\left(x, y^{\prime}, z\right)$ is in $B^{\prime}$. Now, in each row of $R \times C$, $W^{\prime}$ and $B^{\prime}$ contain the same number of symbols, so for any $(x, y, z) \in B$ it follows that $(x, y, z) \in B^{\prime}$ if and only if $\phi(x)=r$ and $\phi(z)=s$. Thus, using the notation from $\S 2.1$, for each $x \in R, \psi_{x}$ setwise fixes the set of symbols contained in columns from $C$.

Next, suppose there is some $(x, y, z) \in B^{\prime}$ and $\left(x^{\prime}, y, z\right) \in W$. Then $\phi(x)=r$ and $\phi(z)=s$ from above. Thus $\phi\left(x^{\prime}\right)=\phi(z)-\phi(y)=r$, so $x^{\prime} \in R$. Thus, for each column $y \in C, \psi_{y}$ setwise fixes the set of symbols in that column contained in rows from $R$. Thus, the restriction of $(W, B)$ to $R \times C$ is a bitrade. Since $(W, B)$ is connected, it contains no entries outside $R \times C$. Finally, apply Lemma 5 .

In a finitely generated abelian group $G$, the torsion subgroup $H$ is the subgroup consisting of all elements of finite order. It is well known that $G / H$ is a direct sum of finitely many copies of $\mathbb{Z}$. Thus by repeated use of Lemma 6 we have:

Corollary 1 Let $(W, B)$ be a connected latin bitrade such that $W$ embeds in a finitely generated abelian group $G$. Then $W$ embeds in the torsion subgroup of $G$.

Corollary 2 Let $W$ be a trade. Then $W$ can be embedded in a finite abelian group iff $W$ can be embedded in a finitely generated abelian group.

Proof The "only if" direction is trivial. For the "if" direction, apply the previous corollary to each connected component of a latin bitrade $(W, B)$, then take the direct sum of the resulting embeddings.

Actually, a large finite cyclic group is locally indistinguishable from $\mathbb{Z}$, so it is not hard to see that Corollary 2 is true for any PLS, not just for trades. It suffices to replace each copy of $\mathbb{Z}$ by $\mathbb{Z}_{m}$ where $m$ exceeds the maximum difference between any two indices that the embedding uses in that copy of $\mathbb{Z}$. However, Corollary 1 does not seem easy to obtain by such an argument, and does not hold for all PLS (to see this, consider any PLS of size 5 embedded in $\mathbb{Z}_{2} \oplus \mathbb{Z}$ ).

A consequence of Corollary 1 is that no trade can be embedded in a torsion-free abelian group. However, it is possible to embed a trade in a torsion-free non-abelian group, as we now show.

Example 3.6 Consider the group $G=\left\langle x, y \mid x^{2} y x^{2}=y, y^{2} x y^{2}=x\right\rangle$. Let $z=x y$ and denote the identity element in $G$ by $\varepsilon$. Let $N$ be the subgroup of $G$ generated by 
$x^{2}, y^{2}$ and $z^{2}$. The following relations are simple consequences of the relations for $G$.

$$
\begin{gathered}
x^{2} y^{2} x^{-2} y^{-2}=x^{2} z^{2} x^{-2} z^{-2}=y^{2} z^{2} y^{-2} z^{-2}=\varepsilon \\
x y^{2}=y^{-2} x, x z^{2}=z^{-2} x, y x^{2}=x^{-2} y, z x^{2}=x^{-2} z, z y^{2}=y^{-2} z, y z^{2}=z^{-2} y .
\end{gathered}
$$

It follows that $N$ is normal and abelian. By a Reidemeister-Schreier rewriting process it can be shown that $N$ is a free abelian group of rank 3. Moreover,

$$
G / N \cong\left\langle x, y \mid x^{2}=y^{2}=(x y)^{2}=\varepsilon\right\rangle \cong \mathbb{Z}_{2} \oplus \mathbb{Z}_{2} .
$$

Each element of $G$ may be written in the form $x^{a} y^{b}\left(x^{2}\right)^{\alpha}\left(y^{2}\right)^{\beta}\left(z^{2}\right)^{\gamma}$ where $a, b \in$ $\{0,1\}$ and $\alpha, \beta, \gamma \in \mathbb{Z}$. Elements of this form may be multiplied using the relations (2). We find that

$$
\begin{aligned}
& \left(x\left(x^{2}\right)^{\alpha}\left(y^{2}\right)^{\beta}\left(z^{2}\right)^{\gamma}\right)^{2}=x^{4 \alpha+2}, \\
& \left(y\left(x^{2}\right)^{\alpha}\left(y^{2}\right)^{\beta}\left(z^{2}\right)^{\gamma}\right)^{2}=y^{4 \beta+2}, \\
& \left(z\left(x^{2}\right)^{\alpha}\left(y^{2}\right)^{\beta}\left(z^{2}\right)^{\gamma}\right)^{2}=z^{4 \gamma+2},
\end{aligned}
$$

each of which is a non-identity element of $N$. Hence the square of every element of $G$ has infinite order, from which it follows that $G$ is torsion-free. Finally, we note that a spherical latin trade embeds in $G$ :

\begin{tabular}{c|cccc}
\hline & $\varepsilon$ & $y x$ & $x$ & $y^{-1} x^{2}$ \\
\hline$\varepsilon$ & $\varepsilon$ & $y x$ & $x$ & $\cdot$ \\
$x^{-1} y^{-1}$ & $x^{-1} y^{-1}$ & $\varepsilon$ & $\cdot$ & $y^{2} x$ \\
$x$ & $x$ & $\cdot$ & $x^{2}$ & $x^{-1} y^{-1}$ \\
$y$ & $\cdot$ & $y^{2} x$ & $y x$ & $x^{2}$
\end{tabular}

\section{The main results}

In this section we prove the main results of the paper, including Theorem 2. We start by outlining some notational conventions which apply throughout the section.

We will use additive notation for groups to emphasise that they are all abelian. Throughout, $W \subseteq R \times C \times S$ is a latin trade of size $n+1$ with a fixed disjoint mate $B$ such that $(W, B)$ is a spherical bitrade. As in $\$ 2.1$, we assume $R, C$ and $S$ are disjoint and we omit any unused rows, columns and symbols. We consider $\mathcal{V}=R \cup C \cup S$ to be a set of commuting indeterminates. From $W$ we define a group $\mathcal{A}_{W}$ with the following presentation:

$$
\mathcal{A}_{W}=\langle\mathcal{V} \mid\{r+c+s=0:(r, c, s) \in W\}\rangle .
$$

Like all groups in this section, it is immediate from its definition that $\mathcal{A}_{W}$ is abelian. We now proceed to deduce some much deeper properties of $\mathcal{A}_{W}$, which will eventually lead to a proof of Theorem 2 . 
We start by considering the standard matrix presentation of $\mathcal{A}_{W}$. We build $\mathcal{M}_{W}$, a $(0,1)$-matrix with rows indexed by $\mathcal{V}$ and columns indexed by $W$, in which there are exactly three 1's per column. The 1's in the column indexed by $(r, c, s) \in W$ appear in the rows indexed by $r, c$ and $s$. Since $(W, B)$ has genus 0 and size $n+1$, we know from (1) that $\mathcal{M}_{W}$ is an $(n+3) \times(n+1)$ matrix. In particular, it has more rows than columns, which means that $\mathcal{A}_{W}$ is infinite. However, we can make use of Lemma 2 to create a related finite group which might help us to prove Theorem 2.

In the triangulation $\mathcal{G}_{W, B}$, we consider the faces corresponding to elements of $W$ to be white and faces corresponding to elements of $B$ to be black. We identify one white triangle as the special white triangle $T_{\Delta}$; say $T_{\Delta}=\left(r_{0}, c_{0}, s_{0}\right)$. We then define a group $\mathcal{A}_{W}^{*}$ with the following presentation:

$$
\mathcal{A}_{W}^{*}=\left\langle\mathcal{V} \mid\{r+c+s=0:(r, c, s) \in W\} \cup\left\{r_{0}=c_{0}=s_{0}=0\right\}\right\rangle .
$$

We also define a corresponding matrix presentation $\mathcal{M}_{W}^{*}$, which is obtained from $\mathcal{M}_{W}$ by deleting the column corresponding to $T_{\Delta}$ and the rows corresponding to $r_{0}$, $c_{0}$ and $s_{0}$. It follows that $\mathcal{M}_{W}^{*}$ is an $n \times n$ matrix. On the face of it, $\mathcal{A}_{W}^{*}$ depends on the choice of $T_{\Delta}$. However, this dependence is superficial, as we show below.

Lemma $7 \mathcal{A}_{W} \cong \mathcal{A}_{W}^{*} \oplus \mathbb{Z} \oplus \mathbb{Z}$.

Proof Consider the homomorphism $\xi: \mathcal{A}_{W} \mapsto \mathcal{A}_{W}^{*} \oplus \mathbb{Z}^{2}$ satisfying $\xi r=(r, 1,0)$, $\xi c=(c, 0,1)$ and $\xi s=(s,-1,-1)$, for every $r \in R, c \in C$ and $s \in S$. It has an inverse satisfying $\xi^{-1}(0,1,0)=r_{0}, \xi^{-1}(0,0,1)=c_{0}, \xi^{-1}(r, 0,0)=r-r_{0}$, $\xi^{-1}(c, 0,0)=c-c_{0}$ and $\xi^{-1}(s, 0,0)=s-s_{0}$, for every $r \in R, c \in C$ and $s \in S$. Thus it provides the required isomorphism.

Corollary 3 Let $\mathcal{A}_{W}^{\#}$ be defined as for $\mathcal{A}_{W}^{*}$ except with a different choice of $T_{\Delta}$. Then $\mathcal{A}_{W}^{\#} \cong \mathcal{A}_{W}^{*}$. Moreover, the isomorphism preserves $r-r^{\prime}$ for all $r, r^{\prime} \in R \subset \mathcal{V}$, the common generating set for $\mathcal{A}_{W}^{\#}$ and $\mathcal{A}_{W}^{*}$.

Proof The proof of Lemma 7 shows that $\mathcal{A}_{W}^{\#} \oplus \mathbb{Z}^{2} \cong \mathcal{A}_{W}^{*} \oplus \mathbb{Z}^{2}$ by an isomorphism that maps $\left(r-r^{\prime}, 0,0\right)$ to $(r, 1,0)-\left(r^{\prime}, 1,0\right)=\left(r-r^{\prime}, 0,0\right)$. The corollary follows.

Of course, the isomorphism in Corollary 3 also preserves $c-c^{\prime}$ and $s-s^{\prime}$ for all $c, c^{\prime} \in C$ and $s, s^{\prime} \in S$. We next prove a lemma about triangles embedded in the plane.

Lemma 8 Let $G$ be a simple plane graph. Suppose that $T_{1}, T_{2}, \ldots, T_{k}$ are distinct triangular faces of $G$, such that every edge of $G$ belongs to precisely one of $T_{1}, \ldots, T_{k}$. Then $G$ has at least $k+2$ vertices. If $k>1$ and $G$ has only $k+2$ vertices then every vertex of $G$ is incident with at least two of $T_{1}, T_{2}, \ldots, T_{k}$.

Proof First suppose that $G$ is connected. We apply Euler's formula to $G$. Let $U_{1}, \ldots, U_{m}$ be the faces of $G$ other than $T_{1}, \ldots, T_{k}$. From the given conditions we know that $G$ has exactly $3 k$ edges and every edge is shared by some $T_{i}$ and some $U_{j}$. Since each $U_{j}$ has at least 3 edges, $3 k \geq 3 m$ and hence $k \geq m$. Now Euler's formula 
tells us the number of vertices in $G$ is $3 k-(k+m)+2 \geq k+2$. If $G$ is not connected, applying the above argument to each connected component gives a similar result.

Moreover, the number of vertices in $G$ equals $k+2$ only if $G$ is connected, $k=m$ and each $U_{j}$ is a triangle. Suppose $T_{i}$ has vertex set $\{X, Y, Z\}$ where $Z$ is not a vertex of any $T_{j}$ for $j \neq i$. Then the $U_{l}$ that contains $Z$ also contains $X$ and $Y$. Assuming $k>1$, this leads to a parallel edge unless $U_{l}$ has more than 3 sides.

Recall that $\mathcal{M}_{W}^{*}$ is a square matrix. We next prove that its permanent is non-zero. Actually, we show something slightly stronger.

Lemma 9 Let $\mathcal{M}_{W}^{\prime}$ denote any matrix obtained from $\mathcal{M}_{W}^{*}$ by setting one of the positive entries to zero. Then $\operatorname{per}\left(\mathcal{M}_{W}^{\prime}\right)>0$.

Proof Suppose by way of contradiction that $\operatorname{per}\left(\mathcal{M}_{W}^{\prime}\right)=0$. Since $\mathcal{M}_{W}^{\prime}$ is a nonnegative matrix, the Frobenius-König Theorem (see, for example, 31.4.1 in [21]) tells us that $\mathcal{M}_{W}^{\prime}$ contains a $(n-k+1) \times k$ submatrix of zeroes for some $1 \leq k \leq n$. Let the column indices of this submatrix be denoted $\Gamma$ and suppose these columns correspond to the triangles $T_{1}, T_{2}, \ldots, T_{k}$ in $\mathcal{G}_{W, B}$. As usual, let $T_{\Delta}$ denote the special triangle.

Applying Lemma 8 to $T_{\Delta}, T_{1}, \ldots, T_{k}$ in the subgraph of $\mathcal{G}_{W, B}$ induced by the edges of these triangles, we find that $T_{1}, T_{2}, \ldots, T_{k}$ between them use at least $k$ vertices that are distinct from the 3 vertices in $T_{\Delta}$. It follows that the columns $\Gamma$ do not have more than $n-k$ zero rows in $\mathcal{M}_{W}^{*}$. Moreover, the condition for equality in Lemma 8 tells us there can only be $n-k$ zero rows if each of the other rows has at least two positive entries. Hence $\mathcal{M}_{W}^{\prime}$ cannot contain $n-k+1$ zero rows in the columns $\Gamma$. This contradicts the choice of $\Gamma$ and proves the Lemma.

Corollary $4 \operatorname{per}\left(\mathcal{M}_{W}^{*}\right) \geq 2$.

The lower bound in Corollary 4 is achieved when $W$ is an intercalate.

An alternate way to state Lemma 9 is that for any given edge in the bipartite graph corresponding to $\mathcal{M}_{W}^{*}$ there is a perfect matching that does not use that edge. The previous sentence is untrue if the word "not" is omitted, as can be demonstrated by taking $W$ to be any trade of size 7 .

There is a wealth of interesting theory surrounding matrices whose permanent and determinant agree up to sign. The interested reader is encouraged to start by consulting [17]. We use one small part of that theory to prove our next result.

Theorem $5\left|\operatorname{det}\left(\mathcal{M}_{W}^{*}\right)\right|=\operatorname{per}\left(\mathcal{M}_{W}^{*}\right)$.

Proof From Corollary 4 there exists at least one positive diagonal $\delta$ of $\mathcal{M}_{W}^{*}$. For a fixed choice of $\delta$ we construct a digraph $D$ with vertices corresponding to the rows of $\mathcal{M}_{W}^{*}$. For each column $\beta$ of $\mathcal{M}_{W}^{*}$, let $\alpha$ be the row of $\mathcal{M}_{W}^{*}$ such that cell $(\alpha, \beta)$ is included in $\delta$. For each row $\alpha^{\prime} \neq \alpha$ that contains a non-zero entry in column $\beta$, we add a directed edge $\left(\alpha, \alpha^{\prime}\right)$ to $D$.

We will show that $D$ contains no dicycles of even length. By Theorem 10 of [17], this gives the required result. (Note that [17] uses a digraph isomorphic to our $D$ 
and a matrix that is equivalent to our $\mathcal{M}_{W}^{*}$ up to a permutation of the rows. Such permutations may change the sign of the determinant, but preserve its magnitude.)

Suppose that there exists a dicycle $\Gamma$ in $D$ of length $l \geq 3$. If we ignore orientation then the vertices and edges of $D$ are a subset of the vertices and edges, respectively, of $\mathcal{G}_{W, B}$. Thus $\mathcal{G}_{W, B}$ induces a planar embedding of $D$. By redrawing $\mathcal{G}_{W, B}$ if necessary, we may assume that the special triangle $T_{\Delta}$ lies outside $\Gamma$. Note that by construction $\Gamma$ cannot contain any vertex from $T_{\Delta}$. We let $G^{\prime}$ be the subgraph of $\mathcal{G}_{W, B}$ which includes $\Gamma$ and any internal faces, vertices and edges. Thus $G^{\prime}$ has one external face with $l$ edges and all other faces are triangles. Suppose $G^{\prime}$ has $v$ vertices, $e$ edges and $f$ faces. Further, suppose that $y$ of the edges of $\Gamma$ are contained in white triangles in $G^{\prime}$. These $y$ edges belong to $y$ different white triangles in $G^{\prime}$, because in any white triangle the oriented edges share the same source, so a dicycle cannot contain more than one of them.

Next, define $f_{B}$ to be the number of black triangles within $G^{\prime}$ and let $f_{W}$ be the number of white triangles within $G^{\prime}$ that do not intersect $\Gamma$. Then, the (vertex, face) pairing given by $\delta$ implies that the number of vertices of $G^{\prime}$ not on $\Gamma$ is equal to $f_{W}$. Thus, $v=l+f_{W}$.

Now, every edge of $G^{\prime}$, other than the $y$ edges on $\Gamma$ which border white triangles, is adjacent to a unique black triangle. Therefore, $e=3 f_{B}+y$ and $f=1+f_{B}+f_{W}+y$. Since $G^{\prime}$ is planar, $f-e+v=2$ by Euler's formula. Combining these equations yields $l=2\left(f_{B}-f_{W}\right)+1$, so in particular $l$ is odd.

Combining Theorem 5 and Corollary 4 we see that $\left|\operatorname{det}\left(\mathcal{M}_{W}^{*}\right)\right| \geq 2$. The theory of presentation matrices (see, for example, [18]) tells us that $\left|\operatorname{det}\left(\mathcal{M}_{W}^{*}\right)\right|$ is the order of $\mathcal{A}_{W}^{*}$ (and that $\operatorname{det}\left(\mathcal{M}_{W}^{*}\right)$ would be 0 if $\mathcal{A}_{W}^{*}$ was infinite). Hence, we have the vital observation that $\mathcal{A}_{W}^{*}$ is non-trivial and finite:

Corollary 5 The abelian group $\mathcal{A}_{W}^{*}$ satisfies $2 \leq\left|\mathcal{A}_{W}^{*}\right|<\frac{\sqrt{2}}{3} 6^{n / 3}$.

Proof The matrix $\mathcal{M}_{W}$ has three 1's in every column. So of the $n$ columns in $\mathcal{M}_{W}^{*}$, there are at least 3 with only two 1's and the remaining columns contain three 1's. Applying the Minc-Brègman Theorem (see, for example, 31.3.5 in [21]) gives the upper bound.

Combined with Lemma 7 we also have:

Corollary $6 \mathcal{A}_{W}$ has free rank exactly two.

We also now know that $\mathcal{M}_{W}^{*}$ is invertible. Our next technical lemma tells us, in effect, that $\left(\mathcal{M}_{W}^{*}\right)^{-1}$ does not have any column which consists of integers.

Lemma 10 Let $\tilde{x}=\left(x_{1}, x_{2}, \ldots, x_{n}\right)^{T}$ and $\tilde{e}_{i}=(0, \ldots, 0,1,0, \ldots, 0)^{T}$ where the sole 1 is in the $i$-th coordinate. The matrix equation $\mathcal{M}_{W}^{*} \tilde{x}=\tilde{e}_{i}$ has no solution in which every $x_{j}$ is an integer. 
Proof Let $M_{i j}$ denote the cofactor of the $(i, j)$-th entry of $\mathcal{M}_{W}^{*}$. From a cofactor expansion for $\operatorname{det}\left(\mathcal{M}_{W}^{*}\right)$ along the $i$-th row we infer that

$$
1=\sum_{j \in \Omega} \frac{M_{i j}}{\operatorname{det}\left(\mathcal{M}_{W}^{*}\right)},
$$

where $\Omega$ is the set of columns in which a 1 appears in row $i$ of $\mathcal{M}_{W}^{*}$. By Lemma 9 there are at least two $j \in \Omega$ for which $M_{i j} \neq 0$. Also, by Theorem 5, the non-zero terms in the sum in (3) are all positive, so they cannot be integers. The result now follows from Cramer's rule, since the unique solution to $\mathcal{M}_{W}^{*} \tilde{x}=\tilde{e}_{i}$ is

$$
x_{j}=\frac{M_{i j}}{\operatorname{det}\left(\mathcal{M}_{W}^{*}\right)}
$$

for each $j$.

Given Corollary 5, our next result will complete the proof of Theorem 2.

Theorem 6 W embeds in $\mathcal{A}_{W}^{*}$.

Proof We need to distinguish between the elements of $\mathcal{V}$ when they are used as coordinates for $W$ as contrasted with when they are considered as generators for $\mathcal{A}_{W}^{*}$. For any $v \in \mathcal{V}$ we will use $\bar{v}$ to denote the group element and $v$ without the bar to designate the coordinate for $W$.

Consider the maps

$$
\begin{aligned}
& I_{1}: R \mapsto \mathcal{A}_{W}^{*} \text { that satisfies } I_{1}\left(r_{0}\right)=0 \text { and } I_{1}\left(r_{i}\right)=\bar{r}_{i} \text { otherwise, } \\
& I_{2}: C \mapsto \mathcal{A}_{W}^{*} \text { that satisfies } I_{2}\left(c_{0}\right)=0 \text { and } I_{2}\left(c_{j}\right)=\bar{c}_{j} \text { otherwise, } \\
& I_{3}: S \mapsto \mathcal{A}_{W}^{*} \text { that satisfies } I_{3}\left(s_{0}\right)=0 \text { and } I_{3}\left(s_{k}\right)=-\bar{s}_{k} \text { otherwise. }
\end{aligned}
$$

For any $\left(r_{i}, c_{j}, s_{k}\right) \in W$, the construction of $\mathcal{A}_{W}^{*}$ ensures that $\bar{r}_{i}+\bar{c}_{j}+\bar{s}_{k}=0$ so that $I_{1}\left(r_{i}\right)+I_{2}\left(c_{j}\right)=I_{3}\left(s_{k}\right)$. It follows that we have an embedding of $W$ in $\mathcal{A}_{W}^{*}$ provided we can show that $I_{1}, I_{2}, I_{3}$ are injections.

We first prove that $\bar{v} \neq 0$ in $\mathcal{A}_{W}^{*}$ for any $v \in \mathcal{V} \backslash\left\{r_{0}, c_{0}, s_{0}\right\}$. Suppose $\bar{v}=0$. Then $\bar{v}$ must be an integer linear combination of the defining relations for $\mathcal{A}_{W}^{*}$. However, Lemma 10 shows this is not the case.

We next argue that $I_{1}(r) \neq I_{1}\left(r^{\prime}\right)$ for distinct $r, r^{\prime} \in R \backslash\left\{r_{0}\right\}$. Suppose $\bar{r}=\bar{r}^{\prime}$. Consider the situation if we had chosen, say, $\left(r^{\prime}, c, s\right)$ as the special white triangle rather than $\left(r_{0}, c_{0}, s_{0}\right)$. By Corollary 3 this would have produced an isomorphic group in which $\bar{r}=\bar{r}^{\prime}=0$, contrary to what we proved above. It follows that $I_{1}$ is injective.

The proofs that $I_{2}$ and $I_{3}$ are injective are similar.

Having shown that spherical trades can always be embedded in a finite abelian group, we pause to consider some properties of the embedding that we have constructed. By definition $\mathcal{A}_{W}$ is the "most free" abelian group that contains the structure of $W$ and is generated by the elements in that structure. However, on the basis of 
Lemma 2 and Corollary 1 , we feel it appropriate to concentrate on $\mathcal{A}_{W}^{*}$, the torsion subgroup of $\mathcal{A}_{W}$. We call the embedding of $W$ in $\mathcal{A}_{W}^{*}$ canonical. Of course, $W$ can be embedded in any group that contains $\mathcal{A}_{W}^{*}$ as a subgroup. It is also plausible that $W$ could be embedded in a group obtained by adding extra relations to $\mathcal{A}_{W}^{*}$, but any such group is a homomorphic image of $\mathcal{A}_{W}^{*}$. In particular, $\mathcal{A}_{W}^{*}$ need not have the minimal order among groups in which $W$ embeds. We will give a concrete example where the minimal and canonical embeddings differ, in Example 5.2.

It is also worth mentioning that the embedding of $W$ in $\mathcal{A}_{W}^{*}$ can be constructed in time polynomial in $|W|$. We may not be able to construct the whole Cayley table for $\mathcal{A}_{W}^{*}$, since that could plausibly be exponentially big (see Corollary 5). However, we can construct that part of the table which contains $W$. It is a simple matter to construct $\mathcal{M}_{W}^{*}$ from $W$. We then find unimodular matrices $U_{1}$ and $U_{2}$ such that $S_{W}=U_{1} \mathcal{M}_{W}^{*} U_{2}$ is the Smith normal form of $\mathcal{M}_{W}^{*}$ (this can be done in polynomial time, see [18]). Now $U_{1}$ encodes the linear relationship between the old and new generators, and hence we can translate between the (isomorphic) groups with presentation matrices $\mathcal{M}_{W}^{*}$ and $S_{W}$ respectively. From this information we get a transparent embedding of $W$ in $\mathcal{A}_{W}^{*}$.

We next return to the order of the group $\mathcal{A}_{W}^{*}$. The lower bound in Corollary 5 is achieved by intercalates. The upper bound is likely to be far from the truth. It is clear from the proof of Theorem 5 that $\mathcal{M}_{W}^{*}$ has a structure very different from the matrices which achieve equality in the Minc-Brègman bound. However, we have not made a concerted effort to improve the upper bound in Corollary 5. It is worth noting, however, that every integer $m \geq 2$ is the order of $\mathcal{A}_{W}^{*}$ for some spherical latin trade $W$. In particular, we can take $W$ to be the first two rows of $\mathbb{Z}_{m}$. It is routine to check that $\mathcal{A}_{W}^{*} \cong \mathbb{Z}_{m}$, and that the minimal and canonical embeddings coincide in this case.

Next, we consider torsion rank, which for abelian groups is defined to be the minimum cardinality of a set of generators for the torsion subgroup. We construct an infinite family of planar latin trades $W$ such that if $W$ embeds in $G$ then the torsion rank of $G$ is at least logarithmic in $|W|$. In particular, the torsion rank of even the minimal embedding for $W$ can be forced to be arbitrarily large.

Lemma 11 For each integer $m \geq 2$, there exists a planar latin bitrade $(W, B)$ of size $8 m$ including distinct rows $r, r_{1}, r_{2}, \ldots, r_{m}$ such that if $W$ is embedded in a finite abelian group $G$ then $2 r=2 r_{1}=\cdots=2 r_{m}$.

Proof Let $W_{0}$ be the latin trade formed from the first two rows of the addition table for $\mathbb{Z}_{m}$, with disjoint mate $W_{0}^{\prime}$ formed by swapping these rows.

By observation, $\left(W_{0}, B_{0}\right)$ is a connected, separated planar latin bitrade of size $2 \mathrm{~m}$. Let $r$ be a row of this latin bitrade. In any planar embedding, there are $m$ black triangles incident with $r$. Let $(r, c, s)$ be one such black triangle.

Introducing rows $r^{\prime}, r_{1}$, columns $c^{\prime}, c^{\prime \prime}$ and symbols $s^{\prime}, s^{\prime \prime}$ which do not occur in $\left(W_{0}, B_{0}\right)$, we form a new latin bitrade $\left(W_{1}, B_{1}\right)$ as follows:

$$
\begin{aligned}
W_{1}= & W_{0} \cup\left\{\left(r, c^{\prime \prime}, s^{\prime}\right),\left(r, c^{\prime}, s^{\prime \prime}\right),\left(r^{\prime}, c, s^{\prime}\right),\left(r^{\prime}, c^{\prime}, s\right),\left(r_{1}, c^{\prime}, s^{\prime}\right),\left(r_{1}, c^{\prime \prime}, s^{\prime \prime}\right)\right\}, \\
B_{1}= & \left(B_{0} \backslash\{(r, c, s)\}\right) \cup\left\{\left(r, c, s^{\prime}\right),\left(r, c^{\prime}, s\right),\left(r^{\prime}, c, s\right),\left(r^{\prime}, c^{\prime}, s^{\prime}\right),\left(r, c^{\prime \prime}, s^{\prime \prime}\right),\right. \\
& \left.\left(r_{1}, c^{\prime \prime}, s^{\prime}\right),\left(r_{1}, c^{\prime}, s^{\prime \prime}\right)\right\} .
\end{aligned}
$$


Fig. 2 The transformation to $\left(W_{1}, B_{1}\right)$

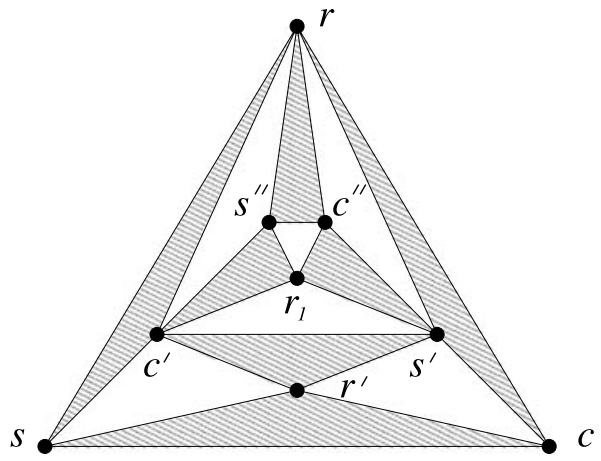

Figure 2 shows the replacement for the black triangle $(r, c, s)$ used to form $\left(W_{1}, B_{1}\right)$. In this diagram the shaded triangles represent black triangles. It is not hard to check that $\left(W_{1}, B_{1}\right)$ is connected and separated.

Moreover, it is clear from Figure 2 that $\left(W_{1}, B_{1}\right)$ is still planar. This fact can also be verified by (1), as we have introduced two new rows, two new columns and two new symbols, with $\left|W_{1}\right|=\left|W_{0}\right|+6$. Thus, $W_{0}$ embeds in a finite abelian group. For any such embedding:

$$
\begin{aligned}
& r+c^{\prime \prime}=s^{\prime}=r_{1}+c^{\prime} \text { and } \\
& r+c^{\prime}=s^{\prime \prime}=r_{1}+c^{\prime \prime} .
\end{aligned}
$$

The above equations imply that $2 r=2 r_{1}$.

By repeating this process for each of the $m$ black triangles from $\left(W_{0}, B_{0}\right)$ that contain row $r$, we obtain a connected, separated planar latin bitrade with size $8 m$ and rows $r, r_{1}, \ldots, r_{m}$ that must satisfy $2 r=2 r_{1}=\cdots=2 r_{m}$ in any group embedding.

Corollary 7 For each integer $m \geq 2$, there exists a planar latin bitrade $(W, B)$ of size $8 m$ such that if $W$ embeds in an abelian group $G$, then $G$ has torsion rank at least $\log _{2}(m+1)$.

Proof For any fixed element $g$ in a finite abelian group $G$ of torsion rank $\rho$, the number of solutions to $2 x=g$ in $G$ is at most $2^{\rho}$. This follows directly from the equivalent statement for cyclic groups. In Lemma 11, $r, r_{1}, \ldots, r_{m}$ are $m+1$ distinct elements of $G$ such that $2 r=2 r_{1}=\cdots=2 r_{m}$.

\section{Embedding Bitrades}

In earlier sections we have considered problems involving embedding a latin trade in a group. However, for any latin trade there is at least one disjoint mate, and it is natural to consider whether the embedding behaviour of the mate is related to the embedding behaviour of the trade. This is the issue considered in this section. 
First we show that for spherical trades there is essentially only one mate that needs to be considered.

Lemma 12 Let $(W, B)$ be a planar latin bitrade. If $\left(W, B^{\prime}\right)$ is a latin bitrade and $B^{\prime} \neq B$, then $\left(W, B^{\prime}\right)$ is neither connected nor separated.

Proof Firstly, suppose that $\left(W, B^{\prime}\right)$ is separated but not connected. Then, there must exist a partition of the rows and columns of this latin bitrade into non-empty sets $R_{1}, R_{2}$ and $C_{1}, C_{2}$, respectively, such that each element of $B^{\prime}$ (and hence $W$ ) lies either in a cell from $R_{1} \times C_{1}$ or a cell from $R_{2} \times C_{2}$. This implies that $(W, B)$ is also disconnected, a contradiction.

Secondly, suppose that $\left(W, B^{\prime}\right)$ is connected but not separated. Then we may transform $\left(W, B^{\prime}\right)$ into a separated latin bitrade of the same size by increasing the number of rows, columns and symbols, as in Example 2.1. This new latin bitrade is connected and separated so has a well-defined genus $g$. However, from (1), $g$ must be strictly less than 0 , the genus of $(W, B)$, a contradiction.

Thirdly, suppose that $\left(W, B^{\prime}\right)$ is both connected and separated. The lemma is trivially true for intercalates, so we assume henceforth there is a row, column or symbol which occurs at least three times. By considering species equivalents if necessary, we lose no generality by assuming that there exists a row $r$ and some $j \geq 2$ such that: $\left\{\left(r, c_{i}, s_{i}\right) \mid 0 \leq i \leq j\right\} \subset W,\left\{\left(r, c_{i}, s_{i+1}\right) \mid 0 \leq i \leq j\right\} \subset B$ (where subscripts are calculated mod $j)$, but $\left(r, c_{0}, s_{1}\right) \notin B^{\prime}$.

We claim that such a circumstance implies that the planar graph $\mathcal{G}_{W, B}$ contains a subdivision of $K_{3,3}$, contradicting Kuratowski's theorem.

Consider the sequence $x(0), x(1), \ldots, x(\ell)$, where $\ell \geq 2, x(0)=0, x(\ell)=1$ and $\left(r, c_{x(i)}, s_{x(i+1)}\right) \in B^{\prime}$ for $0 \leq i \leq \ell-1$. Note that such a sequence exists because $\left(W, B^{\prime}\right)$ is separated. Define $x(m)=\max \{x(i) \mid 1 \leq i \leq \ell-1\}$. We now define our subdivision of $K_{3,3}$. Let the vertices of one colour be $r, c_{0}$ and $c_{x(m)}$ and the vertices of the other colour be $s_{0}, s_{1}$ and $s_{x(m)}$.

Firstly, $r$ is clearly adjacent to $s_{0}, s_{1}$ and $s_{x(m)}$ in $\mathcal{G}_{W, B}$. Next, $\left(r, c_{0}, s_{0}\right) \in W$ implies that $c_{0}$ is adjacent to $s_{0}$. Similarly, $c_{x(m)}$ is adjacent to $s_{x(m)}$. Also $c_{0}$ is adjacent to $s_{1}$ because $\left(r, c_{0}, s_{1}\right) \in B$.

Next, connect $c_{x(m)}$ to $s_{0}$ via a path on the sequence of vertices:

$$
c_{x(m)}, s_{x(m)+1}, c_{x(m)+1}, \ldots, s_{j}, c_{j}, s_{0} .
$$

Connect $c_{0}$ to $s_{x(m)}$ via the path $c_{x(0)}, s_{x(1)}, c_{x(1)}, \ldots, s_{x(m)}$. Finally, connect $c_{x(m)}$ to $s_{1}$ via the path $c_{x(m)}, s_{x(m+1)}, c_{x(m+1)}, \ldots, s_{x(\ell)}$. Observe that each of the above paths are disjoint on internal vertices. We thus have the required subdivision of $K_{3,3}$.

It is important to realise that Lemma 12 does not claim that spherical trades have a unique disjoint mate. To illustrate this point, we exhibit distinct latin bitrades $(W, B)$ and $\left(W, B^{\prime}\right)$ such that $(W, B)$ is connected, separated and planar but $\left(W, B^{\prime}\right)$ is nei- 
ther separated nor connected:

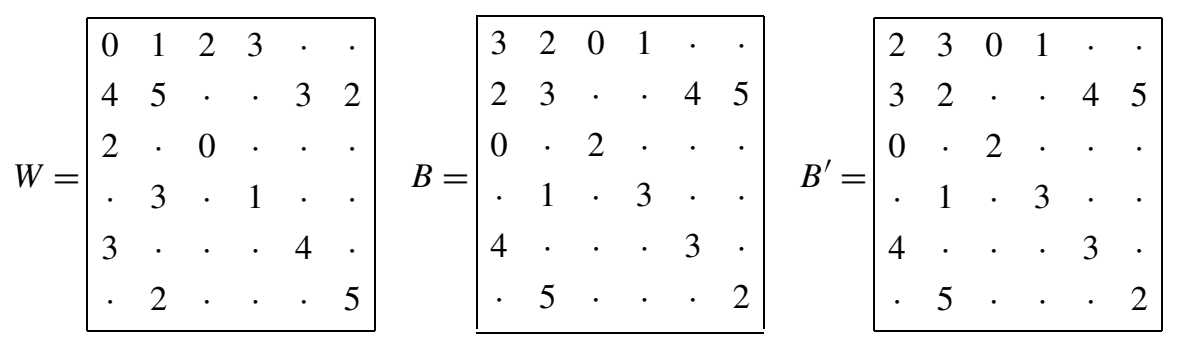

Example 5.1 The smallest connected, separated bitrade $(W, B)$ such that $W$ embeds in a group and $B$ does not embed in any group, is the following example of genus 1 . Let $W$ be the bold entries in the left hand matrix, and $B$ be the entries in the right hand matrix.

\begin{tabular}{|llllll|}
\hline $\mathbf{0}$ & $\mathbf{1}$ & $\mathbf{2}$ & 3 & $\mathbf{4}$ & 5 \\
$\mathbf{1}$ & $\mathbf{2}$ & 3 & 4 & 5 & 0 \\
$\mathbf{2}$ & 3 & $\mathbf{4}$ & 5 & $\mathbf{0}$ & 1 \\
3 & 4 & 5 & 0 & 1 & 2 \\
$\mathbf{4}$ & 5 & $\mathbf{0}$ & 1 & $\mathbf{2}$ & 3 \\
5 & 0 & 1 & 2 & 3 & 4 \\
\hline
\end{tabular}

$W$ embedded in $\mathbb{Z}_{6}$

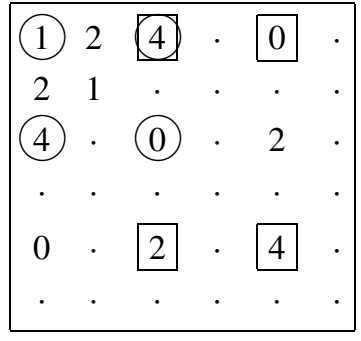

$B$

As shown, $W$ embeds in $\mathcal{A}_{W}^{*}=\mathbb{Z}_{6}$. However, $B$ clearly does not embed in $\mathcal{A}_{B}^{*}=\mathbb{Z}_{3}$. Indeed, it cannot be embedded in any group since it fails the quadrangle criterion, as demonstrated by the two quadrangles marked with $\square$ and $\bigcirc$.

To verify that no smaller bitrade has the claimed property, it suffices to rule out spherical bitrades using Theorem 2 and note that the only two other bitrades of size 11 or smaller are those discussed in Example 3.2.

Example 5.2 For some spherical bitrades $(W, B)$, the smallest group in which $W$ embeds is distinct from the smallest group in which $B$ embeds. The smallest example of this type has size 12. An example of that size is:

\begin{tabular}{|llllll|}
\hline $\mathbf{0}$ & $\mathbf{1}$ & $\mathbf{2}$ & $\mathbf{3}$ & 4 & 5 \\
1 & 2 & 3 & 4 & 5 & 0 \\
$\mathbf{2}$ & $\mathbf{3}$ & $\mathbf{4}$ & 5 & 0 & $\mathbf{1}$ \\
$\mathbf{3}$ & 4 & 5 & $\mathbf{0}$ & 1 & 2 \\
4 & 5 & 0 & 1 & 2 & 3 \\
5 & 0 & $\mathbf{1}$ & 2 & 3 & $\mathbf{4}$ \\
\hline
\end{tabular}

$W$ embedded in $\mathbb{Z}_{6}$

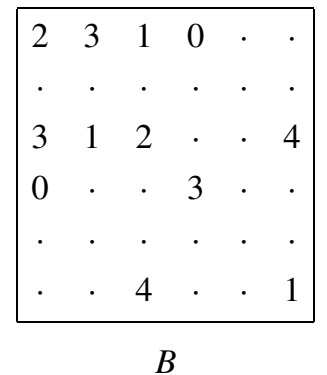

$B$ 
We have demonstrated that $W$ (the bold entries in the left hand square) embeds in $\mathbb{Z}_{6}$. It does not embed in any smaller group, since it uses five distinct columns (ruling out groups of order $<5$ ) and contains a latin subsquare of order 2 (ruling out $\mathbb{Z}_{5}$ ). With a little effort it is possible to show that neither $W$ nor $B$ embeds in $S_{3}$.

We now argue that $B$ cannot be embedded in a cyclic group of any finite order $m$. Along the lines of Example 3.3, we assume $r_{0}=c_{0}=s_{0}=0$ and deduce that $c_{1}=$ $r_{2}=-c_{2}$ and $2 c_{1}=c_{2}$, from which we infer $3 c_{1}=0$. Also $r_{3}=c_{3}=c_{1} / 2, r_{5} \circ c_{5}=$ $c_{2}$ and $r_{2} \circ c_{5}=r_{5} \circ c_{2}$. These last two equations imply $2 r_{5}=r_{2}$. But now we have $c_{1}=r_{2}=2 r_{3}=2 r_{5}$ and $c_{1}=4 c_{1}=2 c_{2}$ which implies that $c_{2}, r_{3}$ and $r_{5}$ cannot be distinct. However, we know $r_{3}$ and $r_{5}$ are distinct and that $c_{2} \neq c_{3}=r_{3}$, so we conclude that $c_{2}=r_{5}$. This is impossible, since then $r_{5} \circ c_{5}=c_{2}=r_{5}=r_{5} \circ c_{0}$, which means that $c_{5}=c_{0}$.

We conclude that $B$ cannot embed in any cyclic group, whereas the smallest group that $W$ embeds in is cyclic. The bitrade $(W, B)$ is the smallest separated, connected example to achieve this property, and also the smallest where the two trades have minimal group embeddings in distinct groups. (Example 3.3 is the only smaller bitrade which does not have the property that both trades embed in the same cyclic group.)

Despite the preceding examples which show that the embedding behaviour is generally different for the two trades in a bitrade, it seems that for spherical trades there is a special relationship between the two trades. If $(W, B)$ is a spherical latin bitrade, then so is $(B, W)$. Thus we may apply the results of $\S 4$ to the black triangles, obtaining an embedding of $B$ into an abelian group $\mathcal{A}_{B}^{*}$. Although the minimal embeddings of $W$ and $B$ may differ, as seen in Example 5.2, we conjecture that their canonical embeddings cannot.

Conjecture 1 If $(W, B)$ is a spherical latin bitrade, then $\mathcal{A}_{W}^{*} \cong \mathcal{A}_{B}^{*}$ and $\mathcal{A}_{W} \cong \mathcal{A}_{B}$.

By Lemma 7, the statements that $\mathcal{A}_{W}^{*} \cong \mathcal{A}_{B}^{*}$ and $\mathcal{A}_{W} \cong \mathcal{A}_{B}$ are equivalent. Conjecture 1 is true for all 72379 species of (unordered) spherical latin bitrades of size up to 19 , as catalogued in [20]. Moreover, the following result also supports Conjecture 1 .

Theorem 7 If $(W, B)$ is a spherical latin bitrade then $\left|\mathcal{A}_{W}^{*}\right|=\left|\mathcal{A}_{B}^{*}\right|$.

Proof To prove this, it suffices to show that $\operatorname{per}\left(\mathcal{M}_{W}^{*}\right)=\operatorname{per}\left(\mathcal{M}_{B}^{*}\right)$. Let $D$ be the dual of the triangulation $\mathcal{G}_{W, B}$. Let $X$ be the set of matchings of size $|W|-1$ in $D$. We count $|X|$ in two different ways. Note that $\operatorname{per}\left(\mathcal{M}_{W}^{*}\right)$ counts bijections between white faces $f$ of $\mathcal{G}_{W, B}$ (other than the special white triangle $T_{\Delta}$ ) and vertices $v_{f}$ of $\mathcal{G}_{W, B}$ (other than those of $T_{\Delta}$ ). We can produce an element of $X$ by matching each $f$ with the black face that is adjacent to $f$ and does not contain $v_{f}$. So, for each vertex $v$ of $D$ corresponding to a white face $f$ of $\mathcal{G}_{W, B}, \operatorname{per}\left(\mathcal{M}_{W}^{*}\right)$ is the number of $(|W|-1)$-matchings that do not cover $v$ (this number is independent of our choice of $v$, by Corollary 3$)$. So $|X|=|W| \operatorname{per}\left(\mathcal{M}_{W}^{*}\right)$. Similarly, considering the black triangles, $|X|=|B| \operatorname{per}\left(\mathcal{M}_{B}^{*}\right)$. As $|W|=|B|$, the result follows. 
(a)

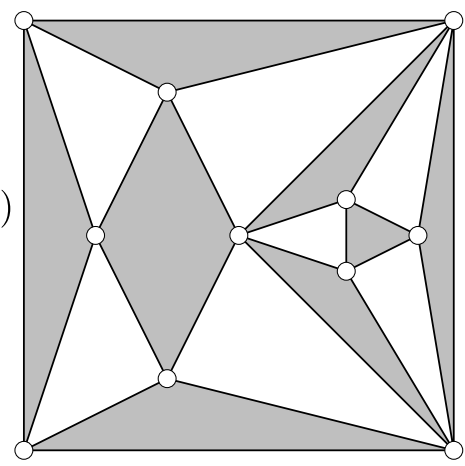

(b)

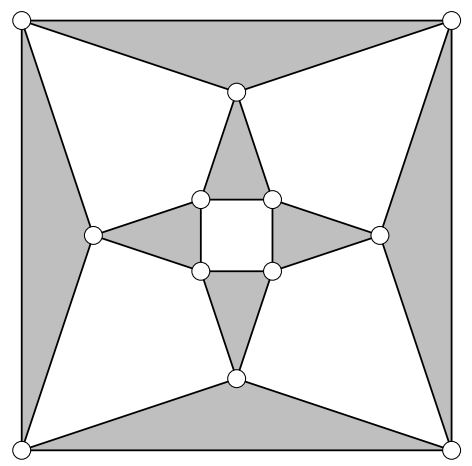

Fig. 3 Two face 2-colourable plane graphs

Several plausible generalisations of Conjecture 1 do not hold. Example 5.1 shows that it does not hold for 2-colourable triangulations of the torus. Also, if we stay in the plane but relax the condition that faces must be triangular, the analogue of Conjecture 1 fails. Figure 3 shows two face 2-colourable plane graphs for which $\mathcal{A}_{W} ¥ \mathcal{A}_{B}$. For graph (a), $\mathcal{A}_{W} \cong \mathbb{Z}_{2} \oplus \mathbb{Z}^{3}$ and $\mathcal{A}_{B} \cong \mathbb{Z}_{4} \oplus \mathbb{Z}^{3}$, while for graph (b), $\mathcal{A}_{W} \cong \mathbb{Z}_{2} \oplus \mathbb{Z}^{6}$ and $\mathcal{A}_{B} \cong \mathbb{Z}^{5}$.

Also, we might drop the condition that indeterminates commute. We could label each vertex of $\mathcal{G}_{W, B}$ with a generator and take relations which say that the product of the vertex labels in a clockwise direction around each white triangle is the identity. We can still, on the basis of Lemma 2, select a special white triangle and set its labels to the identity. Suppose the group so produced is called $\mathcal{N}_{W}^{*}$, and the corresponding group for black triangles is $\mathcal{N}_{B}^{*}$. It is not always the case that $\mathcal{N}_{W}^{*} \cong \mathcal{N}_{B}^{*}$. A concrete example is the bitrade in Example 3.3, for which $\mathcal{N}_{W}^{*}=\left\langle x, y \mid x^{2}=y^{2}, y^{4}=1\right\rangle$ and $\mathcal{N}_{B}^{*}=\left\langle x, y \mid x^{2}=1, y^{2} x y^{2}=x\right\rangle$ (where we use 1 to denote the identity). Let $H_{W}$ and $H_{B}$ be the normal subgroups generated by the squares of all the elements in $\mathcal{N}_{W}^{*}$ and $\mathcal{N}_{B}^{*}$ respectively. Then $\mathcal{N}_{W}^{*} / H_{W} \cong \mathcal{N}_{B}^{*} / H_{B} \cong\langle x, y| x^{2}=y^{2}=$ $\left.(x y)^{2}=1\right\rangle \cong \mathbb{Z}_{2} \oplus \mathbb{Z}_{2}$. However, by a Reidemeister-Schreier rewriting process we find that $H_{W} \cong \mathbb{Z}_{2} \oplus \mathbb{Z} ¥ \mathbb{Z} \oplus \mathbb{Z} \cong H_{B}$, from which it follows that $\mathcal{N}_{W}^{*} ¥ \mathcal{N}_{B}^{*}$.

\section{Closing remarks}

Spherical latin bitrades are equivalent to 2-colourable triangulations of the plane. We have shown (Theorem 6) that for every spherical latin bitrade $(W, B)$ there is a copy of $W$ in a finite abelian group $\mathcal{A}_{W}^{*}$. We showed that $\mathcal{A}_{W}^{*}$ is the torsion subgroup of the group $\mathcal{A}_{W}$ defined by labelling the vertices of the triangulation with commuting indeterminates and adding relations that say the indeterminates around any white triangle add to the identity.

The presentation matrix $\mathcal{M}_{W}^{*}$ for $\mathcal{A}_{W}^{*}$ has the unusual property that $\operatorname{per}\left(\mathcal{M}_{W}^{*}\right)=$ $\left|\operatorname{det}\left(\mathcal{M}_{W}^{*}\right)\right|$ (Theorem 5). The embedding of $W$ in $\mathcal{A}_{W}^{*}$ can be found in polynomial time. Using relations from the black triangles instead of white triangles produces an 
abelian group $\mathcal{A}_{B}^{*}$. We have shown that $\mathcal{A}_{W}^{*}$ and $\mathcal{A}_{B}^{*}$ have the same order (Theorem 7), and we conjecture (Conjecture 1) that they are isomorphic.

Aside from this conjecture a number of interesting open problems remain.

Q1 Which abelian groups arise as $\mathcal{A}_{W}^{*}$ for some spherical latin trade $W$ ? We saw in $\S 4$ that every cyclic group does, and so do at least some groups of large torsion rank. However $\mathbb{Z}_{2} \oplus \mathbb{Z}_{2}$ does not, as can easily be checked by noting that it contains no spherical trades other than intercalates.

Q2 Can the spherical latin trades which embed in cyclic groups be characterised by some property of their triangulations?

Q3 Is there a family of trades $W$ for which $\left|\mathcal{A}_{W}^{*}\right|$ grows exponentially in $|W|$ ?

Q4 What is the best algorithm for determining the minimal embedding for a trade $W$ (that is, the abelian group of minimal order such that $W$ can be embedded in the group)?

Q5 For a trade $W$, how different can the minimal embedding $E_{W}$ be from the canonical embedding in $\mathcal{A}_{W}^{*}$ ? Can $\left|\mathcal{A}_{W}^{*}\right| /\left|E_{W}\right|$ be arbitrarily large? Can the torsion rank for $\mathcal{A}_{W}^{*}$ be arbitrarily much higher than the torsion rank of $E_{W}$ ? (We know from Example 5.2 that it can be higher.)

Q6 Among the $(0,1)$ matrices of order $n$ for which the permanent and determinant agree, what is the largest value that the permanent can take and what structure of matrix achieves that maximum? It might be helpful to restrict the class of matrices by placing conditions on the row or column sums. Answering such questions may improve the upper bound in Corollary 5, which is related to Q3.

There are many other interesting directions in which this research could be taken. For example, one could (a) look for other interesting classes of trades/PLS that embed in groups, (b) find new necessary or sufficient conditions that govern whether a trade/PLS can embed in a group, (c) study groups defined on two face colourable plane graphs other than triangulations, (d) consider infinite triangulations/trades or (e) investigate the relationship between the groups $\mathcal{N}_{W}^{*}$ and $\mathcal{N}_{B}^{*}$ as introduced at the end of the previous section (do they always have the same order?). Example 3.6 shows that $\mathcal{N}_{W}^{*}, \mathcal{N}_{B}^{*}$ will display behaviour that we have not seen in the abelian case.

Acknowledgements The authors are very grateful to Iain Aitchison for interesting discussions, in particular, the tantalising suggestion of connections between Conjecture 1 and knot theory. We are also indebted to the members of the online group-pub-forum, and particularly to Mike Newman, for help with manipulating presentations for non-abelian groups.

We are also very grateful to Aleš Drápal for alerting us after submission to some important and highly relevant work that we had overlooked. Our Lemmas 2, 6 and 7 can be deduced from results in [11] and [12], although the terminology of those papers is somewhat different to the present work. Lemma 4.4 of [13] proves an exponential bound similar to our Corollary 5, but with a better base constant. Indeed, [11-13] anticipate several of the pivotal ideas in our present investigation, including using entries of a partial latin square to create relations in a group, using those relations to write down a $(0,1)$-matrix and investigating conditions under which that matrix has non-zero determinant. Finally, it seems that in [14] our Theorem 2 has been obtained independently using a quite different, and more geometrical argument.

\section{References}

1. Bate, J.A., van Rees, G.H.J.: Minimal and near-minimal critical sets in back circulant latin squares. Australas. J. Comb. 27, 47-61 (2003)

2. Cavenagh, N.J.: The theory and application of latin bitrades: a survey. Math. Slovaca 58, 1-28 (2008) 
3. Cavenagh, N.J.: Embedding 3-homogeneous latin trades into abelian 2-groups. Comment. Math. Univ. Carol. 45, 194-212 (2004)

4. Cavenagh, N.J., Hämäläinen, C., Drápal, A.: Latin bitrades derived from groups. Discrete Math. 308, 6189-6202 (2008)

5. Cavenagh, N.J., Lisoněk, P.: Planar Eulerian triangulations are equivalent to spherical latin bitrades. J. Comb. Theory Ser. A 115, 193-197 (2008)

6. Cooper, J., Donovan, D., Seberry, J.: Latin squares and critical sets of minimal size. Australas. J. Comb. 4, 113-120 (1991)

7. Dénes, J., Keedwell, A.D.: Latin Squares and Their Applications. Akadémiai Kiadó, Budapest (1974)

8. Drápal, A.: On a planar construction of quasigroups. Czechoslov. Math. J. 41, 538-548 (1991)

9. Drápal, A.: Hamming distances of groups and quasi-groups. Discrete Math. 235, 189-197 (2001)

10. Drápal, A., Kepka, T.: Exchangeable partial groupoids I. Acta Univ. Carol. Math. Phys. 24, 57-72 (1983)

11. Drápal, A., Kepka, T.: Group modifications of some partial groupoids. Ann. Discrete Math. 18, 319332 (1983)

12. Drápal, A., Kepka, T.: Group distances of Latin squares. Comment. Math. Univ. Carol. 26, 275-283 (1985)

13. Drápal, A., Kepka, T.: On a distance of groups and Latin squares. Comment. Math. Univ. Carol. 30, 621-626 (1989)

14. Drápal, A., Hämäläinen, C., Kala, V.: Latin bitrades and dissections of equilateral triangles. Preprint

15. Egan, J., Wanless, I.M.: Latin squares with no small odd plexes. J. Comb. Des. 16, 477-492 (2008)

16. Keedwell, A.D.: Critical sets in latin squares and related matters: an update. Util. Math. 65, 97-131 (2004)

17. McCuaig, W.: Pólya's permanent problem. Electron. J. Comb. 11, R79 (2004)

18. Sims, C.C.: Computation with Finitely Presented Groups. Encyclopedia Math. Appl., vol. 48. Cambridge University Press, Cambridge (1994)

19. Suschkewitsch, A.: On a generalization of the associative law. Trans. Am. Math. Soc. 31, 204-214 (1929)

20. Wanless, I.M.: A computer enumeration of small latin trades. Australas. J. Comb. 39, 247-258 (2007)

21. Wanless, I.M.: Permanents. In: Hogben, L. (ed.) Handbook of Linear Algebra. Chapman \& Hall/CRC, Boca Raton (2007)

22. Wanless, I.M.: Personal webpage. http://www.maths.monash.edu.au/ iwanless/

23. Wanless, I.M., Webb, B.S.: The existence of latin squares without orthogonal mates. Des. Codes Cryptogr. 40, 131-135 (2006)

24. Open problems from Workshop on latin trades Prague, 6-10 February 2006. http://www.karlin.mff. cuni.cz/ rozendo/op.html 